\title{
Biallelic mutations in NRROS cause an early onset lethal microgliopathy
}

\author{
Colin Smith ${ }^{1}$ - Barry W. McColl ${ }^{2}$. Anirudh Patir ${ }^{3}$. Jack Barrington ${ }^{1,2}$. Jeremy Armishaw ${ }^{4}$. Antonia Clarke . $^{5}$ \\ Jenny Eaton ${ }^{6}$. Vivienne Hobbs ${ }^{4}$. Sahar Mansour ${ }^{7} \cdot$ Melinda Nolan ${ }^{8} \cdot$ Gillian I. Rice $^{9} \cdot$ Mathieu P. Rodero ${ }^{10}$. \\ Luis Seabra $^{10}$. Carolina Uggenti ${ }^{11}$. John H. Livingston ${ }^{12}$. Leslie R. Bridges ${ }^{13}$. Iona J. M. Jeffrey ${ }^{13}$. \\ Yanick J. Crow ${ }^{10,11}$ (1)
}

Received: 7 October 2019 / Revised: 19 February 2020 / Accepted: 19 February 2020 / Published online: 25 February 2020

(c) The Author(s) 2020

Microglia are tissue-resident macrophages playing essential roles in central nervous system (CNS) development and homeostasis $[14,17]$. The importance of microglia for brain health in humans has been highlighted by the definition of Mendelian disorders associated with dysfunction of microglia-related proteins. These so-called microgliopathies [20] comprise a diverse set of neurological phenotypes including disease due to mutations in $C S F 1 R$ [5, 8, 13], DAP12 and TYROBP/TREM2 [4, 7], USP18 [3, 11, 16, 18], and IRF8 $[2,6]$. Here, we describe a novel early onset lethal encephalopathy due to mutations in the microglial-associated protein NRROS.

Electronic supplementary material The online version of this article (https://doi.org/10.1007/s00401-020-02137-7) contains supplementary material, which is available to authorized users.

Yanick J. Crow

yanickcrow@mac.com

1 Academic Department of Neuropathology, Centre for Clinical Brain Sciences, University of Edinburgh, Edinburgh, UK

2 Centre for Discovery Brain Sciences, UK Dementia Research Institute, University of Edinburgh, Edinburgh, UK

3 The Roslin Institute, University of Edinburgh, Edinburgh, UK

4 Department of Paediatrics, Bay of Plenty District Health Board, Tauranga Hospital, Tauranga, New Zealand

5 Paediatric Neurology Department, St Georges Healthcare NHS Trust, London, UK

6 Genetic Health Service New Zealand, Auckland District Health Board, Auckland City Hospital, Auckland, New Zealand

7 Department of Clinical Genetics, SW Thames Regional Genetics Service, St George's Hospital, University of London, London, UK
We ascertained three patients demonstrating a stereotyped clinical and neuroradiological phenotype (Supplementary material). Patients 1 (P1) and P2, both females, were the first and third children of non-consanguineous parents of Maori descent (family F1442), whilst P3 (family F2382), a male, was the first child of first cousin south Asian parents (Supplementary Figure 1). All three children were born after a normal pregnancy and delivery, and early development was unremarkable. However, in the second year of life, they experienced the onset of refractory seizures and neurodegeneration, leading to death between the ages of 27 and 36 months. Metabolic testing, including for mitochondrial dysfunction, was non-contributory. Neuroimaging initially demonstrated fine calcification at the depths of the cerebral gyri, with normal white matter (Fig. 1). As disease progressed, repeat imaging revealed increased calcification,

8 Department of Paediatric Neurology, Starship Children's Health, Auckland, New Zealand

9 Division of Evolution and Genomic Sciences, School of Biological Sciences, Faculty of Biology, Medicine and Health, University of Manchester, Manchester Academic Health Science Centre, Manchester, UK

10 Laboratory of Neurogenetics and Neuroinflammation, Institut Imagine, Paris, France

11 Centre for Genomic and Experimental Medicine, MRC Institute of Genetics and Molecular Medicine, University of Edinburgh, Edinburgh, UK

12 Department of Paediatric Neurology, Leeds General Infirmary, Leeds, UK

13 Department of Cellular Pathology, St George's University Hospitals NHS Foundation Trust, London, UK 


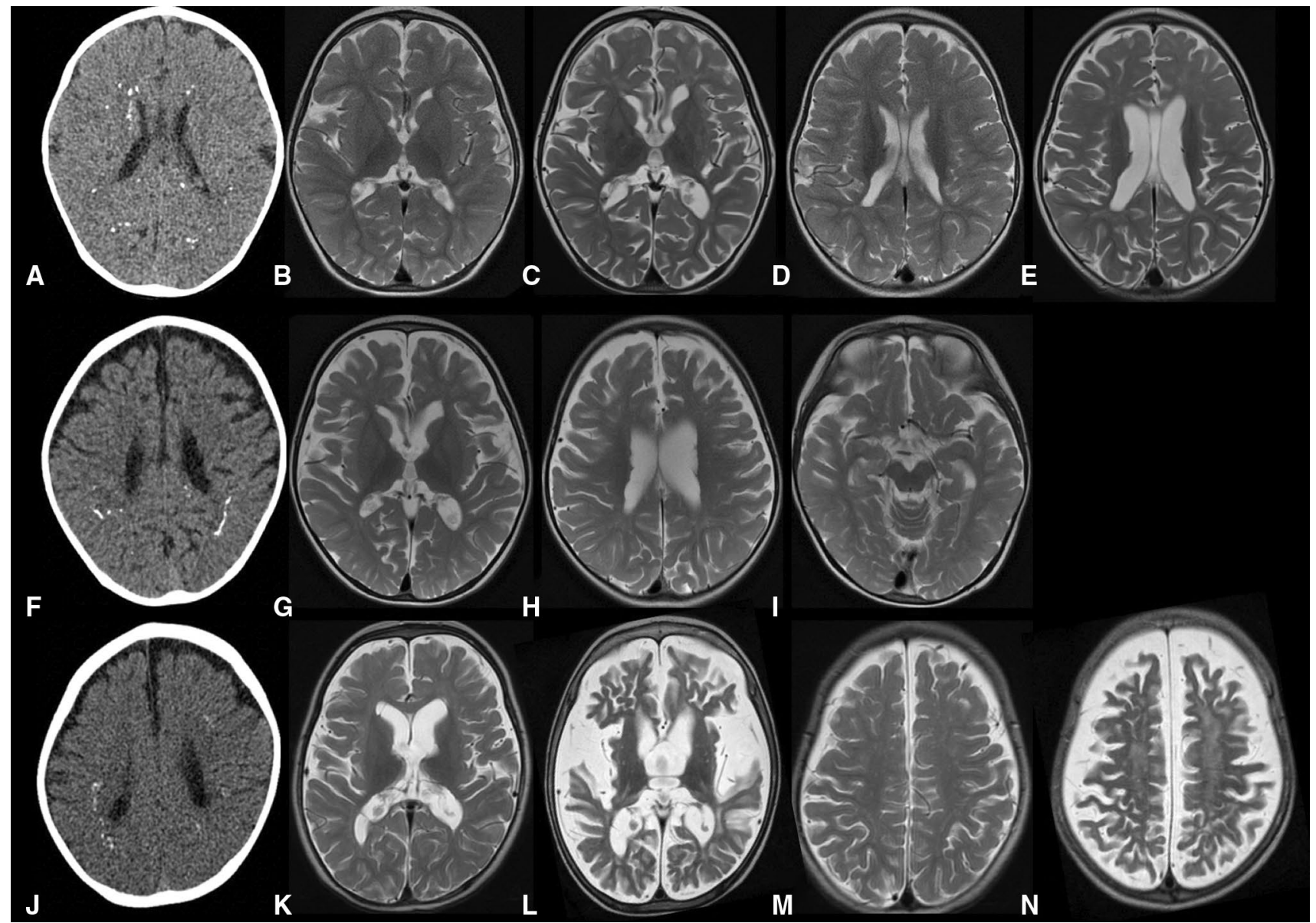

Fig. 1 Neuroimaging of patients from F1442 and F2382. CT images of patient $1(\mathrm{P} 1)$ aged 9 months (a), patient $2(\mathrm{P} 2)$ aged 17 months (f), and patient 3 (P3) aged 14 months (j) demonstrate widespread spot and linear calcification in the deep and sub-cortical white matter. T2-weighted axial MR images of P1 aged 9 months (b, d) and
27 months $(\mathbf{c}, \mathbf{e}), \mathrm{P} 2$ aged 17 months $(\mathbf{g}, \mathbf{h}, \mathbf{i})$, and P3 aged 14 months $(\mathbf{k}, \mathbf{m})$ and 27 months $(\mathbf{l}, \mathbf{n})$. Initial imaging shows mild cerebral volume loss with relatively good myelination. Follow-up shows rapidly progressive cortical and sub-cortical atrophy in P1 and P3. There is diffuse high signal in the white matter in $\mathrm{P} 3(\mathbf{l}, \mathbf{n})$, but not $\mathrm{P} 1$ severe generalized atrophy with ventricular dilatation, and diffuse signal changes in cerebral and cerebellar white matter.

Exome sequencing identified homozygous NRROS variants in the affected children from both families: a c. $1777 \mathrm{C}>\mathrm{T} / \mathrm{p} .\left(\mathrm{Gln} 593^{*}\right)$ and a c.1257del/p.(Gly420AlafsTer14) in F1442 and F2382, respectively. Cellular material was not available from any of the patients. However, both of these variants are predicted to result in a truncated protein, and both are very rare, with the p.(Gln593*) not previously recorded, and the p.(Gly420AlafsTer14) reported on only 1 of 251,438 alleles on gnomAD.

Detailed pathological examination was undertaken on P3, demonstrating abnormalities confined to the CNS. Gross examination indicated a significant cerebral atrophy (Supplementary Figure 2). Histologically, there was both grey and white matter pathology throughout the cerebrum, cerebellum, and brainstem. Focal calcification was noted in the neuropil. There was widespread neuronal loss with reactive gliosis throughout the grey matter (Supplementary Figure 3). The most striking pathological finding was the accumulation of foamy macrophages, predominantly in a perivascular distribution, throughout the white matter, extending from frontal to occipital white matter (Fig. 2a-c), and through cerebellar white matter and descending corticospinal pathways in the basis pontis. These foamy cells immunoreacted with CD68, MHC Class II (CR3/43), and p22phox (Fig. 2c-f), but did not immunoreact with CD163, Iba1, NRROS, CD3, P2Y12, or TMEM1 19 (Supplementary Figure 3). There was reduced myelin basic protein (MBP) expression in the white matter (Fig. 2g) compared to age-matched controls, although there was preservation of U fibers. Occasional axonal spheroids were noted, albeit this was not a prominent feature (Supplementary Figure 3).

We assessed the cellular expression of NRROS, and the mouse homolog Nrros (Lrrc33), in human and mouse brain 


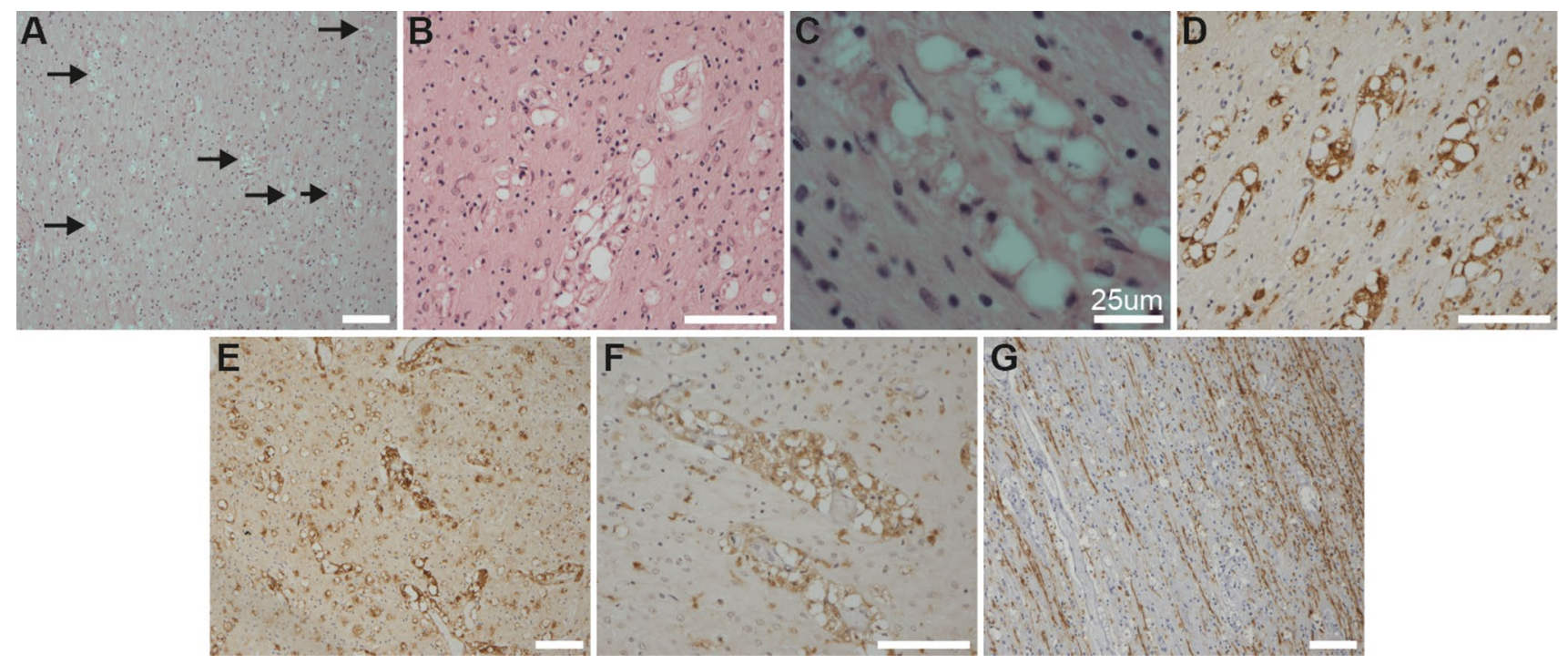

Fig. 2 Post-mortem histological examination of patient 3 (P3). a Low-power view of occipital white matter with accumulation of foamy cells, predominantly in a perivascular location (black arrows for highlighted examples) (H\&E: $\times 100)$; b the perivascular distribution being highlighted at a higher power $(\mathrm{H} \& \mathrm{E} \times 200)$. Granular material is present in the cytoplasm of these foamy cells $(\mathbf{c} H \& \mathrm{E} \times 600)$. The white matter foamy cells were predominantly, but not exclu-

respectively, by mining curated transcriptomic data sets (Supplementary Figure 4). In fresh post-mortem human cortical microglia and brain samples, NRROS was highly expressed in isolated microglia, although less abundantly than established microglial signature genes. The expression of NRROS was enriched > 50-fold in microglia compared to whole brain, indicating that microglia are the major cell type expressing NRROS in human brain parenchyma. A similar pattern of highly enriched expression of $\mathrm{Lrrc} 33 / \mathrm{Nrros}$ was observed in $\mathrm{CD} 11 \mathrm{~b}^{+}$microglia/macrophages in mouse brain relative to brain extracts, and in microglia versus other parenchymal cell types. Comparison of parenchymal microglia with CNS perivascular macrophages (PVMs) showed significantly greater expression in the latter.

The clinical features observed in our patients recapitulate those in mice with Nrros/Lrrc33 deficiency. Nrros-/-mice exhibited progressive neurological decline, including motor defects and abnormal locomotor activity, from age $2-3$ months and death by 6 months of age [15, 21]. Neuropathology in these mouse models includes neuronal loss, demyelination, axonal pathology, astrogliosis, and the increased presence of foamy macrophages, all of which were seen in our case. Of note, there was no indication of immune-mediated inflammation in our case or either of these mouse models.

NRROS is a leucine-rich repeat containing transmembrane protein localized to the endoplasmic reticulum, and preferentially expressed in myeloid cells. Reported sively, limited to a perivascular distribution and expressed CD68 (d CD68 IHC $\times 200)$, HLA DR/DP/DQ (e CR3/43 IHC × 100), and p22PHOX (f p22phox IHC $\times 200$ ). There was partial preservation of myelin in sub-cortical $U$ fibers, but loss of myelin when assessed by myelin basic protein expression in deeper white matter ( $\mathrm{g}$ myelin basic protein $\mathrm{IHC} \times 100)$

functions include the regulation of reactive oxygen species (ROS) production through control of NOX2 stability [12], responsiveness of Toll-like receptor signaling [19], and processing/activation of transforming growth factor (TGF)- $\beta$ via physical interactions with the latent complex $[10,15]$. NRROS expression is restricted to microglia within the CNS parenchymal compartment in humans and mice. The present case showed disruption to the distribution, density, and cell morphology of IBA1 cells alongside loss of P2Y12 staining and weak TMEM119 immunoreactivity, indicative of marked parenchymal microglial abnormalities. Both Nrros-/- mouse studies observed a loss of homeostatic gene expression profile which included suppression of P2ry 12 and Tmem1 19 expression, and a shift towards a phenotype resembling PVMs [15, 21]. Although Nrros is expressed in peripheral mononuclear cells, a series of crosses and bone marrow transplant experiments showed a negligible contribution of peripheral macrophages to the onset of the Nrros-/- phenotype [21]. Of note, selective deletion of Nrros in microglia during pregnancy indicated a cell-intrinsic role for NRROS. In contrast, Nrros deletion induced in 3 -week-old mice did not cause neuropathological changes or neurological abnormalities [21], implying that NRROS is important during microglial establishment at embryonic/ postnatal stages, but may be dispensable for maintenance of adult microglia. Functions of NRROS proposed above, notably in ROS and TGF $\beta$ regulation, may be important in disease pathogenesis. p22phox was markedly up-regulated in 
PVMs in our case, suggesting that an absence of functional NRROS may result in increased p22phox-NOX2 binding, with the potential for increased superoxide radical formation. However, a cross of Nrros-/- and Cybb-/- (encoding NOX2) mice did not rescue the Nrros-/- phenotype [21]. Mice with CNS or microglial-restricted disruption during development of other key nodes in the TGF $\beta$ activation/ signaling pathway, including deletion of $\alpha \mathrm{V} \beta 8$ integrin or TGFBR2 [1], develop highly similar pathological, microglial, and neurological abnormalities to Nrros-/- mice. Moreover, human TGF $\beta 1$ loss-of-function mutations causing early onset leukoencephalopathy were described recently [9].

Taken together with the mouse data, our findings indicate that NRROS is indispensable in controlling the early development of a homeostatic microglial population and/or its ongoing preservation in the postnatal brain, thereby suggesting a loss of NRROS function as a novel microgliopathy in humans.

Acknowledgements YJC acknowledges a state subsidy managed by the National Research Agency (France) under the 'Investments for the Future' program bearing the reference ANR-10-IAHU-01 and the MSDAvenir fund (DEVO-DECODE Project). YJC and CS acknowledge the RS MacDonald Seed-corn Fund. The DDD study presents independent research commissioned by the Health Innovation Challenge Fund (Grant number HICF-1009-003), a parallel funding partnership between the Wellcome Trust and the Department of Health, and the Wellcome Trust Sanger Institute (Grant number WT098051). BWM acknowledges funding from the UK Dementia Research Institute and Medical Research Council (MR/L003384/1, MR/R001316/1). YJC thanks David Fitzpatrick for advice.

Open Access This article is licensed under a Creative Commons Attribution 4.0 International License, which permits use, sharing, adaptation, distribution and reproduction in any medium or format, as long as you give appropriate credit to the original author(s) and the source, provide a link to the Creative Commons licence, and indicate if changes were made. The images or other third party material in this article are included in the article's Creative Commons licence, unless indicated otherwise in a credit line to the material. If material is not included in the article's Creative Commons licence and your intended use is not permitted by statutory regulation or exceeds the permitted use, you will need to obtain permission directly from the copyright holder. To view a copy of this licence, visit http://creativecommons.org/licenses/by/4.0/.

\section{References}

1. Arnold TD, Lizama CO, Cautivo KM, Santander N, Lin L, Qiu H et al (2019) Impaired $\alpha \mathrm{V} \beta 8$ and TGF $\beta$ signaling lead to microglial dysmaturation and neuromotor dysfunction. J Exp Med 216:900 915. https://doi.org/10.1084/jem.20181290

2. Bigley V, Maisuria S, Cytlak U, Jardine L, Care MA, Green K et al (2018) Biallelic interferon regulatory factor 8 mutation: a complex immunodeficiency syndrome with dendritic cell deficiency, monocytopenia, and immune dysregulation. J Allergy Clin Immunol 141:2234-2248. https://doi.org/10.1016/j.jaci.2017.08.044
3. Goldmann T, Zeller N, Raasch J, Kierdorf K, Frenzel K, Ketscher $\mathrm{L}$ et al (2015) USP18 lack in microglia causes destructive interferonopathy of the mouse brain. EMBO J 34:1612-1629. https:// doi.org/10.15252/embj.201490791

4. Guerreiro RJ, Lohmann E, Bras JM, Gibbs JR, Rohrer JD, Gurunlian $\mathrm{N}$ et al (2013) Using exome sequencing to reveal mutations in TREM2 presenting as a frontotemporal dementia-like syndrome without bone involvement. JAMA Neurol 70:78-84. https://doi. org/10.1001/jamaneurol.2013.579

5. Guo L, Bertola DR, Takanohashi A, Saito A, Segawa Y, Yokota $\mathrm{T}$ et al (2019) Bi-allelic CSF1R mutations cause skeletal dysplasia of dysosteosclerosis-pyle disease spectrum and degenerative encephalopathy with brain malformation. Am J Hum Genet 104:925-935. https://doi.org/10.1016/j.ajhg.2019.03.004

6. Hambleton S, Salem S, Bustamante J, Bigley V, Boisson-Dupuis $S$, Azevedo J et al (2011) IRF8 mutations and human dendriticcell immunodeficiency. N Engl J Med 365:127-138. https://doi. org/10.1056/NEJMoa1100066

7. Konishi H, Kiyama H (2018) Microglial TREM2/DAP12 signaling: a double-edged sword in neural diseases. Front Cell Neurosci 12:206. https://doi.org/10.3389/fncel.2018.00206

8. Konno T, Kasanuki K, Ikeuchi T, Dickson DW, Wszolek ZK (2018) CSF1R-related leukoencephalopathy: a major player in primary microgliopathies. Neurology 91:1092-1104. https://doi. org/10.1212/WNL.0000000000006642

9. Kotlarz D, Marquardt B, Barøy T, Lee WS, Konnikova L, Hollizeck $S$ et al (2018) Human TGF- $\beta 1$ deficiency causes severe inflammatory bowel disease and encephalopathy. Nat Genet 50:344-348. https://doi.org/10.1038/s41588-018-0063-6

10. Ma W, Qin Y, Chapuy B, Lu C (2019) LRRC33 is a novel binding and potential regulating protein of TGF- $\beta 1$ function in human acute myeloid leukemia cells. PLoS One 14:e0213482. https://doi. org/10.1371/journal.pone.0213482

11. Meuwissen ME, Schot R, Buta S, Oudesluijs G, Tinschert S, Speer SD et al (2016) Human USP18 deficiency underlies type 1 interferonopathy leading to severe pseudo-TORCH syndrome. J Exp Med 213:1163-1174. https://doi.org/10.1084/jem.20151529

12. Noubade R, Wong K, Ota N, Rutz S, Eidenschenk C, Valdez PA et al (2014) NRROS negatively regulates reactive oxygen species during host defence and autoimmunity. Nature 509:235-239. https ://doi.org/10.1038/nature13152

13. Oosterhof N, Chang IJ, Karimiani EG, Kuil LE, Jensen DM, Daza R et al (2019) Homozygous mutations in CSF1R cause a pediatric-onset leukoencephalopathy and can result in congenital absence of microglia. Am J Hum Genet 104:936-947. https://doi. org/10.1016/j.ajhg.2019.03.010

14. Prinz M, Priller J (2014) Microglia and brain macrophages in the molecular age: from origin to neuropsychiatric disease. Nat Rev Neurosci 15:300-312. https://doi.org/10.1038/nrn3722

15. Qin Y, Garrison BS, Ma W, Wang R, Jiang A, Li J et al (2018) A milieu molecule for TGF- $\beta$ required for microglia function in the nervous system. Cell 174:156-171.e16. https://doi.org/10.1016/j. cell.2018.05.027

16. Rodero MP, Crow YJ (2016) Type I interferon-mediated monogenic autoinflammation: the type I interferonopathies, a conceptual overview. J Exp Med 213:2527-2538. https://doi.org/10.1084/ jem.20161596

17. Salter MW, Stevens B (2017) Microglia emerge as central players in brain disease. Nat Med 23:1018-1027. https://doi.org/10.1038/ nm.4397

18. Schwabenland M, Mossad O, Peres AG, Kessler F, Maron FJM, Harsan LA et al (2019) Loss of USP18 in microglia induces white matter pathology. Acta Neuropathol Commun 7:106. https://doi. org/10.1186/s40478-019-0757-8

19. Su X, Mei S, Liang X, Wang S, Liu J, Zhang Y et al (2014) Epigenetically modulated LRRC33 acts as a negative physiological 
regulator for multiple Toll-like receptors. J Leukoc Biol 96:17-26. https://doi.org/10.1189/jlb.0813457

20. van der Knaap MS, Bugiani M (2017) Leukodystrophies: a proposed classification system based on pathological changes and pathogenetic mechanisms. Acta neuropathol 134:351-382. https ://doi.org/10.1007/s00401-017-1739-1

21. Wong K, Noubade R, Manzanillo P, Ota N, Foreman O, Hackney JA et al (2017) Mice deficient in NRROS show abnormal microglial development and neurological disorders. Nat Immunol 18:633-641. https://doi.org/10.1038/ni.3743

Publisher's Note Springer Nature remains neutral with regard to jurisdictional claims in published maps and institutional affiliations. 